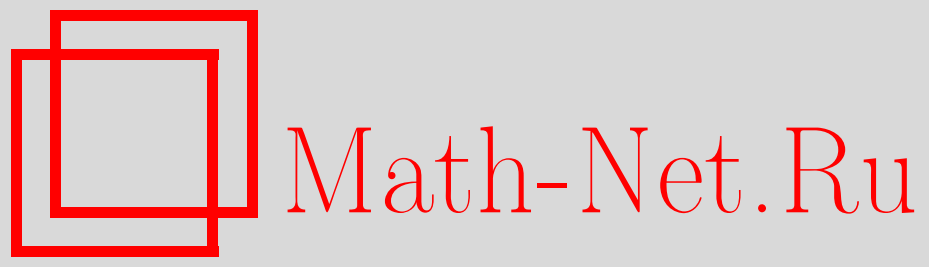

A. Abrams, H. Landau, Z. Landau, J. Pommersheim, E. Zaslow, An iterated random function with Lipschitz number one, Теория вероятн. и ее примен., 2002, том 47, выпуск 2, 286-300

DOI: https://doi.org/10.4213/tvp3648

Использование Общероссийского математического портала MathNet.Ru подразумевает, что вы прочитали и согласны с пользовательским соглашением

http: //www . mathnet.ru/rus/agreement

Параметры загрузки:

IP: 34.229 .108 .108

26 апреля 2023 г., 15:14:21

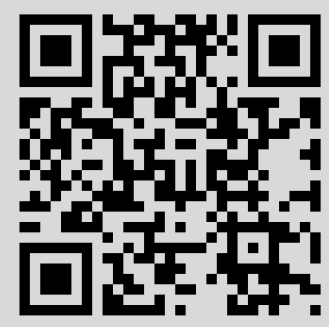



POMMERSHEIM J.***, ZASLOW E. ${ }^{\dagger}$

\title{
AN ITERATED RANDOM FUNCTION WITH LIPSCHITZ NUMBER ONE
}

\begin{abstract}
Рассмотрим множество функций $f_{\theta}(x)=|\theta-x|$ на $\mathbf{R}$. Определим марковский процесс, который стартует в точке $x_{0} \in \mathbf{R}$ и далее определяется по формуле $x_{k+1}=f_{\theta_{k+1}}\left(x_{k}\right)$, где $\theta_{k+1}$ имеет фиксированное ограниченное распределение $\mu$ на $\mathbf{R}^{+}$. Мы доказываем гипотезу Ж. Летака (G. Letac) о том, что если $\mu$ не сосредоточено на решетке, то марковский процесс имеет единственное стационарное распределение $\pi_{\mu}$ и любое распределение сходится при итерациях к $\pi_{\mu}$ (в слабой-* топологии). Мы также приводим оценку для скорости сходимости в частном случае, когда $\mu$ сосредоточено в двух точках. Эта техника может применяться для изучения других марковских процессов, переходные функции которых имеют число Липшица, равное единице.
\end{abstract}

Ключевые слова и фразы: марковский процесс, итерации случайных функций, стационарное распределение.

\section{Introduction}

In their recent paper [1], P. Diaconis and D. Freedman describe a general method for studying Markov chains. This consists of viewing a state transition of the chain as the action of a function chosen at random from a family of state transformations; the Markov process can then be expressed as an iteration of these functions. When the state space is a metric space, e.g., $\mathbf{R}$, they show that if the functions exhibit a certain average contractivity (described in terms of Lipschitz numbers), then running the iteration backward rather than forward produces geometric convergence to a unique

${ }^{*}$ Department of Mathematics, University of Georgia, Athens, GA, 30602 U.S.A.; e-mail: abrams@math.uga.edu; url:www.math.uga.edu/ abrams

${ }^{* *}$ Mathematical Sciences Research Institute, 1000 Centennial Drive, Berkeley, CA, 94720, U.S.A.; e-mail: landau@math.berkeley.edu

${ }^{* * *}$ Department of Mathematics, Pomona College, 610 N. College Ave, Claremont, CA, 91711, U.S.A.; e-mail: jpommersheim@pomona.edu

${ }^{\dagger}$ Department of Mathematics, Northwestern University, 2033 Sheridan Road, Evanston, IL, 60208, U.S.A.; url: www.math.northwestern.edu/ zaslow 
stationary distribution for the chain. Many applications of this idea are developed.

An example of a Markov process not immediately amenable to this method, originally proposed for study by Letac [4], is defined on the nonnegative reals $\mathbf{R}^{+}=[0, \infty)$ by starting with a point $x_{0} \in \mathbf{R}^{+}$and for $k=0,1, \ldots$, setting

$$
x_{k+1}=f_{\theta_{k+1}}\left(x_{k}\right) \equiv\left|\theta_{k+1}-x_{k}\right|,
$$

where the $\theta_{k} \in \mathbf{R}^{+}$are chosen independently from a fixed distribution $\mu$ (on $\mathbf{R}^{+}$). If we choose the starting point $x_{0}$ from a distribution $\pi_{0}$, then the above Markov process defines a distribution $\pi_{n}$ for $x_{n}$.

We will consider the case that $\mu$ has finite expectation; it is known (e.g., $[2$, p. 208]) that in this case the distribution

$$
\pi_{\mu}(x)=\frac{1}{E(\theta)} \int_{0}^{x} \operatorname{Pr}\{\theta>y\} d y
$$

is a stationary probability distribution for this process (where $\theta$ is chosen according to the distribution $\mu$ and $E(\theta)$ is its expectation). G. Letac conjectured in [4] that if $\mu$ is not supported on a lattice (that is, the set of integer multiples of a fixed real number), then $\pi_{\mu}$ is the unique stationary distribution, and that the distributions $\pi_{n}$ of $x_{n}$ should converge to $\pi_{\mu}$ in the weak-* topology. Both these conjectures would follow from Theorem 1 of [1] if the $f_{\theta}$ 's had Lipschitz numbers less than one (on average).

In this paper we prove Letac's conjecture for compactly supported $\mu$.

Theorem 3.1. Let $\mu$ be a distribution on a bounded interval $Y \subset \mathbf{R}^{+}$, with $\mu$ not supported on a lattice. Then $\pi_{\mu}$ is the unique stationary distribution for the process (1). Moreover, if $x_{0}$ is chosen according to an initial distribution $\pi_{0}$ then the distributions $\pi_{n}$ of $x_{n}$ converge to $\pi_{\mu}$ in the weak-* topology.

We prove this theorem in Sections 2 and 3, and in Sections 4 and 5 we analyze more carefully the special case of $\mu$ supported on just two points. In this case we provide a second independent proof of Letac's conjecture, and we also obtain a bound on the rate of convergence of the distributions $\pi_{n}$. Our hope is that the techniques will be useful for the study of other Markov processes, where the transition functions have Lipschitz number one.

After completing this work the authors learned of a paper by J. Mattingly [5] addressing related questions; that paper obtains results similar to ours.

Let $\mu$ be a probability measure on a space $Y$ and let $\left\{f_{\theta}: \theta \in Y\right\}$ be a set of maps from a metric space $X$ into itself. The iterated random function induced by $\mu$ is the Markov process of repeatedly picking $\theta \in Y$ according to $\mu$ and then applying $f_{\theta}$. For $x \in X$, and $\Theta=\left(\theta_{1}, \theta_{2}, \ldots\right)$ with $\theta_{i} \in Y$, 
let $B_{n}(\Theta, x)$ be the $n$-th backward iterate of $x$ and $F_{n}(\Theta, x)$ the $n$-th forward iterate of $x$; that is,

$$
B_{n}(\Theta, x)=f_{\theta_{1}} \circ \cdots \circ f_{\theta_{n}}(x), \quad F_{n}(\Theta, x)=f_{\theta_{n}} \circ \cdots \circ f_{\theta_{1}}(x) .
$$

Letac observed that for fixed $n$ and variable $\Theta$, the distributions of $B_{n}(\Theta, x)$ and $F_{n}(\Theta, x)$ are identical; yet for fixed $\Theta$, as $n$ tends to infinity the sequences $B_{n}(\Theta, x)$ and $F_{n}(\Theta, x)$ behave quite differently. He used this to prove the following general principle.

Letac's principle [4]. If the backward iterates $B_{n}(\Theta, x)$ converge for almost all $\Theta$ to a limit $B(\Theta)$ which is independent of the starting point $x \in X$, then the distribution $\pi_{\mu}$ on $X$ induced ${ }^{1)}$ by the map $\Theta \mapsto B(\Theta)$ is the unique stationary distribution for the iterated random function induced by $\mu$. Moreover, if $x_{0}$ is chosen according to an initial distribution $\pi_{0}$, then the distributions $\pi_{n}$ of $x_{n}$ converge to $\pi_{\mu}$ in the weak-* topology.

In Section 2 we prove the following general result which implies the hypotheses of Letac's principle. Note that this theorem requires checking a property of only the forward iterates.

Theorem 2.1. Consider a probability measure $\mu$ on a space $Y$ and $a$ set of functions $\left\{f_{\theta}: \theta \in Y\right\}$ on a complete metric space $X$. Suppose there exists a subset $S \subset X$ such that

(i) $f_{\theta}(S) \subset S$ almost surely for $\theta$ picked from $\mu$, and

(ii) $\lim _{n \rightarrow \infty} \operatorname{diam}\left(F_{n}(\Theta, S)\right)=0$ almost surely, where $\Theta=\left(\theta_{1}, \theta_{2}, \ldots\right)$ and the $\theta_{i}$ are picked independently from $\mu$.

Then the backward iterates applied to an element of $S$ converge almost surely and the limit is independent of the starting point (in $S$ ).

In Section 3 we establish the hypotheses of Theorem 2.1, completing our proof of Letac's conjecture.

Our second proof of Letac's conjecture applies only in the special case that $\mu$ is uniform on a two-point set $\{\alpha, \beta\}$; however the argument is independent of Letac's principle. Our method is to analyze the iterated random function by studying the orbit of a point; we begin this study in Section 3 and continue in more detail in Section 4 . This technique leads to a bound in Section 5 (Theorem 5.1) on the rate of convergence of the distributions $\pi_{n}$ in this case.

\section{A general result}

In this section we prove a general theorem which lets us conclude that the backward iterates converge by looking only at the forward iterates.

1) For any set $S \subset X$, the induced probability distribution $\pi_{\mu}(S)$ is the measure of the set $\{\Theta \mid B(\Theta) \in S\}$. 
Let $\mu$ be a probability measure on a set $Y$ and $\left\{f_{\theta}: \theta \in Y\right\}$ a set of functions from a complete metric space $X$ into itself. Denote by $\Theta=\left(\theta_{1}, \theta_{2}, \ldots\right)$ an element of $Y^{\infty}=Y \times Y \times \cdots$ and by $\mu^{\infty}=\mu \times \mu \times \cdots$ the infinite product measure on $Y^{\infty}$. The following lemma is used in the proof of Theorem 2.1 to move from considering backward iterates to considering forward iterates.

Lemma 2.1. Let $\Gamma \subset Y^{\infty}$, and let $k_{1}, \ldots, k_{n}$ be a finite set of distinct indices. Let

$$
\Gamma^{\prime}=\left\{\Theta^{\prime} \text { : there exists } a \Theta \in \Gamma \text { such that } \theta_{k_{j}}^{\prime}=\theta_{j}, j=1, \ldots, n\right\} \text {, }
$$

where $\Theta=\left(\theta_{1}, \theta_{2}, \ldots\right)$ and $\Theta^{\prime}=\left(\theta_{1}^{\prime}, \theta_{2}^{\prime}, \ldots\right)$. Then $\mu^{\infty}(\Gamma) \leqslant \mu^{\infty}\left(\Gamma^{\prime}\right)$.

P r o of. Let $\sigma$ be any finite permutation of $\mathbf{N}$ with $\sigma(j)=k_{j}$ for $j=1, \ldots, n$. (A finite permutation is one with $\sigma(j)=j$ for all $j$ sufficiently large.) Since $\mu^{\infty}$ is invariant under the action of finite permutations, we have $\mu^{\infty}(\Gamma)=\mu^{\infty}(\sigma(\Gamma))$. But $\sigma(\Gamma) \subset \Gamma^{\prime}$, so the result follows.

For $S \subset X$, set $B_{n}(\Theta, S)=\left\{B_{n}(\Theta, x): x \in S\right\}$ and $F_{n}(\Theta, S)=$ $\left\{F_{n}(\Theta, x): x \in S\right\}$. Let $\operatorname{diam}(S)=\sup _{x, y \in S} d(x, y)$ be the diameter of $S$ (where $d$ denotes the distance function on $X$ ).

Theorem 2.1. Suppose there exists a subset $S$ of $X$ such that

(i) $f_{\theta}(S) \subset S$ for almost every $\theta$ picked from $\mu$,

(ii) $\lim _{n \rightarrow \infty} \operatorname{diam}\left(F_{n}(\Theta, S)\right)=0$ for almost every $\Theta$ picked from $\mu^{\infty}$.

Then for all $x \in S$, the backward iterates $B_{n}(\Theta, x)$ converge for almost all $\Theta$, and the limit is independent of $x($ in $S)$.

P r o of. Fix $\varepsilon>0$. Set $\Gamma_{N}^{i}=\left\{\Theta: \operatorname{diam}\left(F_{n}(\Theta, S)\right)<2^{-i}\right.$ for all $n \geqslant N\}$. Since $\Gamma_{N}^{i} \subset \Gamma_{N+1}^{i}$ and $\mu^{\infty}\left(\bigcup_{n} \Gamma_{n}^{i}\right)=1$ (by hypothesis (ii)), there exists $N(i)$ such that $\mu^{\infty}\left(\Gamma_{N(i)}^{i}\right)>1-\varepsilon 2^{-i}$. Applying Lemma 2.1 to the set $\Gamma=\Gamma_{N(i)}^{i}$ with $k_{j}=N(i)+1-j, j=1, \ldots, N(i)$, we see that the measure of the set $\Lambda_{N(i)}^{i}=\left\{\Theta: \operatorname{diam}\left(B_{N(i)}(\Theta, S)\right)<2^{-i}\right\}$ is greater than or equal to $\mu^{\infty}\left(\Gamma_{N(i)}^{i}\right)>1-\varepsilon 2^{-i}$. Hypothesis (i) implies that for $n \geqslant N$, $B_{n}(\Theta, S) \subset B_{N}(\Theta, S)$ for almost all $\Theta$; hence

$$
\begin{gathered}
\mu^{\infty}\left\{\Theta: \operatorname{diam}\left(B_{n}(\Theta, S)\right)<2^{-i} \text { and } B_{n}(\Theta, S) \subset B_{N(i)}(\Theta, S)\right. \\
\text { for all } n \geqslant N(i)\}=\mu^{\infty}\left(\Lambda_{N(i)}^{i}\right)>1-\varepsilon 2^{-i} .
\end{gathered}
$$

Letting

$$
\begin{gathered}
\Omega=\bigcap_{i}\left\{\Theta: \operatorname{diam}\left(B_{n}(\Theta, S)\right)<2^{-i} \text { and } B_{n}(\Theta, S) \subset B_{N(i)}(\Theta, S)\right. \\
\text { for all } n \geqslant N(i)\},
\end{gathered}
$$

(3) implies $\mu^{\infty}(\Omega) \geqslant 1-\sum_{i} \varepsilon 2^{-i}=1-\varepsilon$. It follows from the definition of $\Omega$ that the $\left\{B_{n}(\Theta, x)\right\}_{n}$ are Cauchy sequences (i.e., the backward iterates converge) for every $x \in S, \Theta \in \Omega$. Furthermore, since for 
$x, y \in S,\left|B_{n}(\Theta, x)-B_{n}(\Theta, y)\right| \leqslant \operatorname{diam}\left(B_{n}(\Theta, S)\right)$, we see that for $\Theta \in \Omega$, $\lim B_{n}(\Theta, x)=\lim B_{n}(\Theta, y)$. Thus we have shown that on the set $\Omega$ of measure at least $1-\varepsilon$, the backward iterates applied to an element of $S$ converge to a limit independent of the starting point. Since $\varepsilon$ is arbitrary, the result follows.

\section{Proof of Letac's conjecture}

We now return to the iterated random function described by equation (1). In this section, we consider $\mu$ supported on a bounded subset $Y=[0, b]$ of $\mathbf{R}^{+}$. Setting $S=\left[0, b^{\prime}\right]$ for any $b^{\prime} \geqslant b$, it is clear that condition (i) of Theorem 2.1 is satisfied. In the rest of this section we show that $S$ and $\left\{f_{\theta}: \theta \in[0, b]\right\}$ satisfy condition (ii) of Theorem 2.1 reformulation, and then apply the conclusion of Theorem 2.1 to complete the proof of Theorem 3.1.

The argument showing that $S$ and $\left\{f_{\theta}: \theta \in[0, b]\right\}$ satisfy condition (ii) of Theorem 2.1 can be summarized as follows: on an interval $I, f_{\theta}$ acts in one of two ways. If $\theta \notin I$, then $f_{\theta}$ translates $I$; whereas if $\theta \in I$, then $f_{\theta}$ translates and «folds» (and in particular shrinks) $I$. Furthermore, for $\theta$ near the center of $I, f_{\theta}$ will shrink $I$ by about half. Condition (ii) follows from showing that the process defined by equation (1) (for suitable $\mu$ ) almost halves an interval infinitely many times. The key fact we will use is that the orbit of a point is dense.

3.1. The orbit of a point. In this section we discuss the orbit of a point $x$ under iterates of the two functions $f_{\alpha}(x)=|\alpha-x|$ and $f_{\beta}(x)=|\beta-x|$, where $0<\alpha<\beta$. We use the standard notation $x \bmod y$ to mean the unique number $z, 0 \leqslant z<y$, such that $z-x$ is an integer multiple of $y$. Let $x \in[0, \beta]$.

The orbit $\mathscr{O}_{x}$ of $x$ is the set of points $y$ such that $y=f_{\theta_{n}} \circ \cdots \circ f_{\theta_{1}}(x)$ for some $n$, with each $\theta_{i} \in\{\alpha, \beta\}$.

Lemma 3.1. For each $x \in[0, \beta]$, we have $\mathscr{O}_{x}=\{n \alpha+\varepsilon x \bmod \beta: n \in \mathbf{Z}$, $\varepsilon= \pm 1\}$.

P r o o f. Let $S_{x}=\{n \alpha+\varepsilon x \bmod \beta: n \in \mathbf{Z}, \varepsilon= \pm 1\}$. As shorthand, we represent the real number $n \alpha+\varepsilon x \bmod \beta$ by the ordered pair $(n, \varepsilon) \in$ $\mathbf{Z} \times\{ \pm 1\}$. Note that $f_{\beta}((n, \varepsilon))=\beta-(n \alpha+\varepsilon x \bmod \beta)=(-n,-\varepsilon)$. Also,

$$
f_{\alpha}((n, \varepsilon))= \begin{cases}(n-1, \varepsilon) & \text { if }(n, \varepsilon) \geqslant \alpha, \\ \alpha-(n \alpha+\varepsilon x \bmod \beta)=(-(n-1),-\varepsilon) & \text { if }(n, \varepsilon)<\alpha ;\end{cases}
$$

thus the set $S_{x}$ is closed under $f_{\theta}$ for each $\theta \in\{\alpha, \beta\}$ and we have $\mathscr{O}_{x} \subseteq S_{x}$.

To show $S_{x} \subseteq \mathscr{O}_{x}$, it suffices to show that

(1) $(n, \varepsilon) \in \mathscr{O}_{x} \Longrightarrow(-n,-\varepsilon) \in \mathscr{O}_{x}$, and

(2) $(n, \varepsilon) \in \mathscr{O}_{x} \Longrightarrow(n-1, \varepsilon) \in \mathscr{O}_{x}$.

The first claim follows by applying $f_{\beta}$. The second follows by applying $f_{\alpha}$ if $n \alpha+\varepsilon x \bmod \beta \geqslant \alpha$, or by first applying $f_{\alpha}$ and then applying $f_{\beta}$ if $n \alpha+\varepsilon x \bmod \beta<\alpha$. 
Corollary 3.1. Let $0<\alpha<\beta$. If $\alpha / \beta$ is irrational then the orbit of any point under iteration of the functions $f_{\alpha}, f_{\beta}$ is dense in $[0, \beta]$. If $\alpha / \beta=$ $p / q$ with $p, q \in \mathbf{N}$ relatively prime, then the orbit of any point intersects each closed subinterval of $[0, \beta]$ of length at least $\beta / q$.

$\mathrm{Pr}$ o o f. If $\alpha / \beta$ is irrational then the set $\{n \alpha \bmod \beta\}$ is dense in $[0, \beta]$, so the orbit of a point is also dense in $[0, \beta]$. If $\alpha / \beta=p / q$ then the orbit of $x$ contains the set $\{n \alpha+\varepsilon x \bmod \beta\}=\{k \beta / g+x \bmod \beta \mid k=0, \ldots, q-1\}$ which intersects each closed subinterval of $[0, \beta]$ of length at least $\beta / q$.

3.2. Shrinking intervals. Denote the length of an interval $I$ by $\|I\|$.

The following lemma gives a criterion for recognizing when the interval $f_{\theta_{n}} \circ \cdots \circ f_{\theta_{1}}(I)$ is no bigger than about half the size of $I$.

Lemma 3.2. Let $\Theta=\left(\theta_{1}, \theta_{2}, \ldots\right)$, with $\theta_{i} \in \mathbf{R}^{+}$, and let $I \subset \mathbf{R}^{+}$be a bounded interval with midpoint $m$. Fix $\delta_{1}, \delta_{2}>0$. If some $x$ with $|x-m|<\delta_{1}$ satisfies $F_{n}(\Theta, x)<\delta_{2}$, then

$$
\left\|F_{n}(\Theta, I)\right\| \leqslant \frac{1}{2}\|I\|+\delta_{1}+\delta_{2} .
$$

P r o o f. The functions $f_{\theta}$ have Lipschitz number 1 , so for all $y \in I$, we have

$$
\left|F_{n}(\Theta, x)-F_{n}(\Theta, y)\right| \leqslant|x-y| \leqslant \frac{1}{2}\|I\|+\delta_{1} .
$$

Since $F_{n}(\Theta, x) \leqslant \delta_{2}$, it follows that $F_{n}(\Theta, y) \leqslant \frac{1}{2}\|I\|+\delta_{1}+\delta_{2}$. Also $F_{n}(\Theta, y) \geqslant 0$, so the result follows.

We say that $x \in \mathbf{R}^{+}$is a point of increase for the probability distribution $\mu$ if $\mu(I)>0$ for every open interval $I$ containing $x$. The nonzero real numbers $\alpha$ and $\beta$ are irrationally related if $\alpha / \beta$ is irrational.

Lemma 3.3. Let $\mu$ be a probability distribution supported on a bounded interval $Y \subset \mathbf{R}^{+}$. Suppose that $\mu$ is not supported on a lattice. Then for any interval $I \subset \mathbf{R}^{+}$and any $\varepsilon>0$, there exist $N=N(I, \varepsilon)$ and intervals $J_{1}, \ldots, J_{N}$ with $\mu\left(J_{i}\right)>0$, such that for all $\left(\theta_{1}, \ldots, \theta_{N}\right) \in J_{1} \times \cdots \times J_{N}$,

$$
\left\|f_{\theta_{N}} \circ \cdots \circ f_{\theta_{1}}(I)\right\|<\varepsilon .
$$

$\mathrm{P}$ r o o f. We first assert that for any interval $K$, and for any $r>0$, there exist intervals $J_{1}, \ldots, J_{n}$ with $\mu\left(J_{i}\right)>0$ such that for all $\left(\theta_{1}, \ldots, \theta_{n}\right) \in$ $J_{1} \times \cdots \times J_{n}$

$$
\left\|f_{\theta_{n}} \circ \cdots \circ f_{\theta_{1}}(I)\right\|<\left(\frac{1}{2}+r\right)\|K\| .
$$

The result then follows by repeating this shrinking process.

To prove the assertion, we will first construct the midpoints $m_{i}$ of the intervals $J_{i}$. We consider two cases; as $\mu$ is not supported on a lattice, either (a) $\mu$ has two irrationally related points of increase, or (b) $\mu$ has an infinite set of points of increase which are pairwise rationally related. 
Let $m$ be the midpoint of the interval $K$.

First, suppose (a) holds. Let $\alpha<\beta$ be two irrationally related points of increase for $\mu$. By Corollary 3.1 the orbit of $m$ under iteration of the functions $f_{\alpha}$ and $f_{\beta}$ is dense in $[0, \beta]$; thus there exists a sequence $m_{1}, \ldots, m_{n}$ with each $m_{i}$ equal to $\alpha$ or $\beta$ such that

$$
f_{m_{n}} \circ \cdots \circ f_{m_{1}}(m)<\frac{r}{2}\|K\| .
$$

Now suppose (b) holds. In this case we can find points of increase $\alpha, \beta$ of $\mu$ with $\alpha / \beta=p / q$, with $p, q \in \mathbf{N}$ relatively prime and $q$ arbitrarily large. (Note that we cannot simultaneously guarantee that $\alpha<\beta$.) Find such $\alpha, \beta$ with $q>2 \beta /(r\|K\|)$. By Corollary 3.1, the orbit of $m$ under the functions $f_{\alpha}$ and $f_{\beta}$ intersects the closed interval $[0, \beta / q] \subset[0, r\|K\| / 2]$. Thus again there exist $m_{1}, \ldots, m_{n}$ with each $m_{i}$ equal to $\alpha$ or $\beta$ such that (4) holds.

We have now defined the $m_{i}$ in each case. Set $J_{i}$ to be an interval with midpoint $m_{i}$ and length no bigger than $r\|K\| /(2 n)$. Note that $J_{i}$ has positive measure because every $m_{i}$ is a point of increase of $\mu$.

To verify the claim, note that for any $\left(\theta_{1}, \ldots, \theta_{n}\right) \in J_{1} \times \cdots \times J_{n}$, we have

$$
f_{\theta_{n}} \circ \cdots \circ f_{\theta_{1}}(m)<\frac{r}{2}\|K\|+\frac{n r}{2 n}\|K\|=r\|K\|,
$$

since each $f_{\theta}$ has Lipschitz number 1 . Thus we can apply the previous lemma with $x=m$, any $\delta_{1}>0$, and $\delta_{2}=r\|K\|$.

Theorem 3.1. Let $\mu$ be a distribution on a bounded interval $Y \subset \mathbf{R}^{+}$, with $\mu$ not supported on a lattice. Then $\pi_{\mu}$ is the unique stationary distribution for the process (1). Moreover, if $x_{0}$ is chosen according to an initial distribution $\pi_{0}$ then the distributions $\pi_{n}$ of $x_{n}$ converge to $\pi_{\mu}$ in the weak-* topology.

P r o of. We will show that $\left\{f_{\theta}: \theta \in Y\right\}, S=\left[0, b^{\prime}\right], b^{\prime} \geqslant b$, satisfy conditions (i) and (ii) of Theorem 2.1. Consequently, the backward iterates converge independent of a starting point chosen in $\bigcup_{b^{\prime} \geqslant b}\left[0, b^{\prime}\right]=\mathbf{R}^{+}$and the application of Letac's principle completes the proof.

It is clear that $S$ satisfies condition (i) of Theorem 2.1 for all $\Theta \in Y^{\infty}$. To establish (ii), fix $\varepsilon>0$. By Lemma 3.3 (with $I=\left[0, b^{\prime}\right]$ ), there exist intervals $J_{1}, \ldots, J_{N}$ such that

$$
\left\|f_{\theta_{N}} \circ \cdots \circ f_{\theta_{1}}(I)\right\|<\varepsilon \text { for all }\left(\theta_{1}, \ldots, \theta_{N}\right) \in J_{1} \times \cdots \times J_{N} .
$$

As the $f_{\theta}$ 's do not lengthen intervals, we have $\|F(\Theta, S)\|<\varepsilon$ for any $\Theta=$ $\left(\theta_{1}, \theta_{2}, \ldots\right)$ such that for some $K, \theta_{K+i} \in J_{i}$ for each $i=1, \ldots, N$. Since each $\mu\left(J_{i}\right)>0$, this occurs with probability 1 . Since $\varepsilon$ was arbitrary, condition (ii) of Theorem 2.1 is satisfied. 


\section{Special case: two-point distributions}

Our second proof of Theorem 3.1 relies on a detailed understanding of the orbits $\mathscr{O}_{x}$. In this section suppose $\theta$ is chosen from the uniform distribution $\mu$ on the set $\{\alpha, \beta\}$ with $0<\alpha<\beta$; i.e., $\operatorname{Pr}(\theta=\alpha)=\operatorname{Pr}(\theta=\beta)=\frac{1}{2}$. By scaling, we may assume $\beta=1$; we do so for the remainder of the paper. We use the notation $\langle x\rangle$ to denote the number $x \bmod 1$.

4.1. The graph of an orbit. Recall Lemma 3.1, and note that the proof of Lemma 3.1 (with $0<\alpha<\beta=1$ ) reveals the complete structure of the orbit of $x \leqslant 1$. From now on assume $\alpha$ is irrational. Notice that the numbers $\langle n \alpha+\varepsilon x\rangle$ are distinct unless $x$ is in the orbit of 0 (which is also the orbit of 1 and $\alpha$ ), the orbit of $\frac{1}{2}$, the orbit of $\alpha / 2$, or the orbit of $(1+\alpha) / 2$. These four orbits we call singular; other orbits are generic.

To visualize the orbit $\mathscr{O}_{x}$, we associate to each point $x$ a labeled directed graph $G_{x}$, as follows. The vertex set of $G_{x}$ is $\mathscr{O}_{x}$, with the vertex $\langle n \alpha+\varepsilon x\rangle$ labeled with the ordered pair $(n, \varepsilon)$. This label is unique if $\mathscr{O}_{x}$ is generic. A directed edge goes from $(n, \varepsilon)$ to $\left(n^{\prime}, \varepsilon^{\prime}\right)$ if there exists a $\theta$ such that $f_{\theta}(\langle n \alpha+\varepsilon x\rangle)=\left\langle n^{\prime} \alpha+\varepsilon^{\prime} x\right\rangle$; the proof of Lemma 3.1 tells us exactly, where to put these edges.

Note that if $x^{\prime} \in \mathscr{O}_{x}$ then $G_{x^{\prime}}$ looks exactly like $G_{x}$ but with different labels. Also note that if $\mathscr{O}_{x}$ is singular then a vertex of $G_{x}$ will have multiple labels due to coincidences among the numbers $\langle n \alpha+\varepsilon x\rangle$; e.g., a vertex labeled $(n, \varepsilon)$ in $G_{0}$ is also labeled $(n,-\varepsilon)$.

(a)

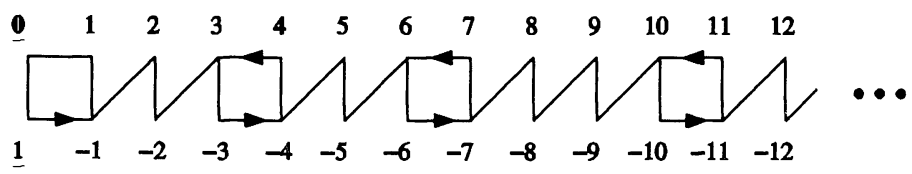

(b)

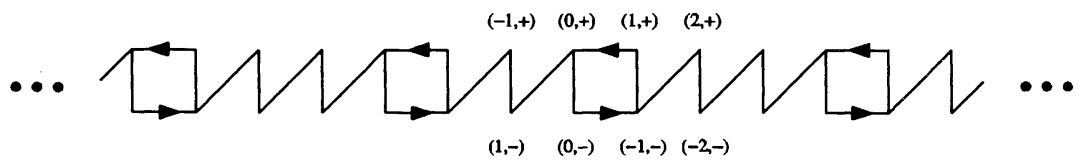

Figure 1. Two orbits for $\alpha=1 / \sqrt{2}>\frac{1}{2}$. The orbit of $x=0.2$ is generic (a), and the orbit of $x=0$ is singular (b). 6 In the orbit of 0 , vertices are labeled only with $n$, since $\varepsilon$ does not matter. The boldface labels $\underline{0}$ and $\underline{1}$ refer to the actual numbers 0 and 1 .

We illustrate the case $\alpha=1 / \sqrt{2}$. Figure 1 (a) shows the (generic) graph $G_{0.2}$, and Figure $1(\mathrm{~b})$ shows the (singular) graph $G_{0}$. In the singular orbit we have consolidated the pair of labels $(n, \varepsilon)$ and $(n,-\varepsilon)$ into the single label $n$. Both graphs have been drawn so that the vertical edges represent an application of $f_{1}$ and the horizontal and diagonal edges represent 
an application of $f_{\alpha}$. An undirected edge indicates two oppositely oriented edges. Note that each vertex has out-degree two, since $\theta$ has two possible values.

The large-scale properties of the graphs $G_{x}$ (for general irrational $\alpha$ ) are important as well. Suppose for a moment that $\alpha>\frac{1}{2}$. There are three types of vertices in a generic graph (see Figure 1):

- small, where $\langle n \alpha+\varepsilon x\rangle<1-\alpha$;

- medium, where $1-\alpha<\langle n \alpha+\varepsilon x\rangle<\alpha$; and

- large, where $\langle n \alpha+\varepsilon x\rangle>\alpha$.

The large vertices have in-degree one, and appear in $G_{x}$ as upper-right and lower-left corners of boxes. The small vertices have in-degree three, and form the other corners of boxes. The medium vertices are the remaining ones, which are not part of any box. The fact that $\langle n \alpha\rangle$ is uniformly distributed mod 1 implies that the ratio of the numbers of small: medium: large vertices is $1-\alpha: 2 \alpha-1: 1-\alpha$. If we write $\alpha=q(1-\alpha)+r$ with $q$ a positive integer and $0<r<1-\alpha$, then the number of diagonal edges between consecutive boxes is either $q$ or $q+1$. These numbers occur in a pattern depending on $x$ but always in the ratio $(1-\alpha-r): r$. For $\alpha=1 / \sqrt{2}$ (Figure 1) we see that there are either 2 or 3 diagonal edges between consecutive boxes, this number being 2 about $58.6 \%$ of the time (since 2 occurs exactly $(3-4 \alpha) /(3 \alpha-2)=$ $\sqrt{2}$ times as often 3$)$.

(a)

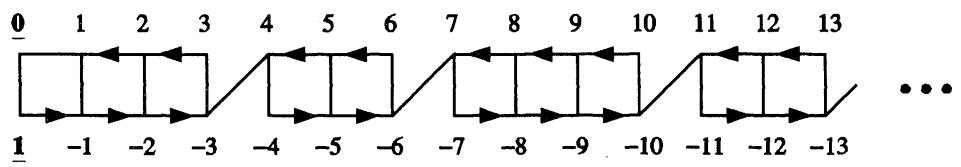

(b)

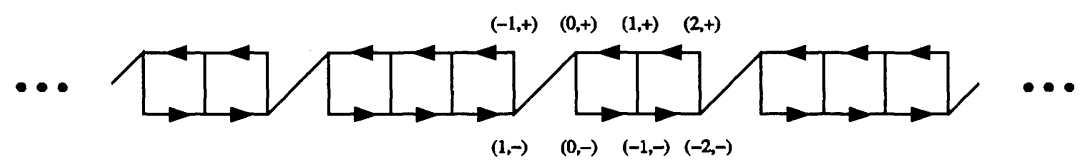

Figure 2. Two orbits for $\alpha=1-1 / \sqrt{2}<\frac{1}{2}$. Again, the orbit of $x=0.2$ is generic (a) and the orbit of $x=0$ is singular (b).

In the case $\alpha<\frac{1}{2}$, the graphs are similar, but we exchange the roles of $\alpha$ and $1-\alpha$ in the definitions of small, medium, and large. The graphs $G_{x}$ in this case have more boxes and fewer diagonal edges. See Figure 2, where $\alpha=1-1 / \sqrt{2}$. The large vertices are the upper-right and lower-left corners of the rectangular strings of boxes; the small vertices are the other corners of the rectangles; and the medium vertices are those vertices which are part 
of two distinct boxes. The ratio of numbers of small : medium : large vertices is $\alpha: 1-2 \alpha: \alpha$. This time writing $1-\alpha=q \alpha+r$, the number of boxes between consecutive diagonal edges is again either $q$ or $q+1$, and these occur in the ratio $(\alpha-r): r$.

Incidentally if $\alpha$ is rational then the graphs of orbits are finite, but they exhibit many properties analogous to the infinite case.

4.2. Uniqueness of stationary distribution. Assume $\alpha \in(0,1)$ is irrational and $\mu$ is uniform on $\{\alpha, 1\}$. By (2), the stationary distribution $\pi_{\mu}$ is given by

$$
\pi_{\mu}(x)= \begin{cases}\frac{2 x}{1+\alpha} & \text { if } 0 \leqslant x \leqslant \alpha \\ \frac{x+\alpha}{1+\alpha} & \text { if } \quad \alpha \leqslant x \leqslant 1\end{cases}
$$

Let $\pi$ be any stationary distribution for the iterated random function (1) induced by $\mu$. We will think of $\pi$ as its distribution function, namely a rightcontinuous increasing function $\pi:[0,1] \rightarrow[0,1]$; thus $\pi(x)$ is the amount of mass which is concentrated on $\{y: y \leqslant x\}$.

We will show that the values of $\pi$ are determined by $\alpha$; the values we compute agree with the corresponding values for $\pi_{\mu}$.

Being right-continuous, $\pi$ is determined by its values on a dense set; with the aid of Figures 1(a) and 2(a) we shall calculate $\pi(x)$ for $x \in \mathscr{O}_{0}=\{\langle n \alpha\rangle\}$.

By stationarity, the mass of $\pi$ is concentrated on $[0,1]$; thus $\pi(1)=1$. We begin by establishing that $\pi$ cannot have an atom (that is, a jump discontinuity) at $\langle n \alpha\rangle$ for any $n \in \mathbf{Z}$.

Lemma 4.1. Let $0<\alpha<1$ be irrational, let $\mu$ be uniform on $\{\alpha, 1\}$, and let $\pi$ be a stationary distribution for the associated iterated random function. Then $\pi(0)=0$ and $\pi$ is continuous at $x=\langle n \alpha\rangle, n= \pm 1, \pm 2, \ldots$

P r o o f. Suppose $\frac{1}{2}<\alpha<1$. If a distribution $\pi_{0}$ has an atom of mass $m$ at 0 , then (referring to the graph $G_{0}$ ) we see that $\pi_{1}$ will have an atom of mass $m / 2$ at 1 , since the only way for $x_{1}$ to equal 1 is if $x_{0}=0$ and $\theta_{1}=1$. Thus if $\pi=\pi_{0}$ is stationary and $\pi$ has an atom of mass $m$ at 0 , then $\pi$ also has an atom of mass $m / 2$ at 1 .

Likewise, iterating the random function shows that at each vertex of $G_{0}$ there must be an atom having mass equal to half the sum of the masses of the atoms at all incoming vertices. It follows that there must be an atom of mass $3 m / 2$ at $\alpha$, an atom of mass $2 m$ at $\langle-\alpha\rangle$, and thereafter an atom of mass $2 m$ at every small and medium vertex and an atom of mass $m$ at every large vertex.

As the total mass of a distribution must be 1 , it is impossible to have $m>0$. 
Thus $\pi(0)=0$. We now note that if $\pi$ were not continuous at some point $\langle n \alpha\rangle$, then $\pi$ would have an atom there; then by the same reasoning $\pi$ would have an atom at 0 , a contradiction.

The case $0<\alpha<\frac{1}{2}$ is similar (see, e.g., Figure 2(a)). Here an atom of mass $m$ at 0 implies an atom of mass $m / 2$ at 1 , an atom of mass $3 m / 2$ at $\alpha$, and an atom of mass $m$ at $1-\alpha$, and thereafter atoms of mass $2 m$ at each small vertex and of mass $m$ at each medium or large vertex. As before, $m$ must be 0 , and the result follows.

We are now able to compute the values of $\pi(x)$ for $x \in \mathscr{O}_{0}$, using a technique similar to that employed in the lemma. Observe that the stationarity of $\pi$ is equivalent to the condition that for each $x \in[0,1]$ we have

$$
\begin{aligned}
\pi(x) & =\sum_{\theta \in\{1, \alpha\}} \mu(\theta)\left(\sum_{\left\{y: f_{\theta}(y)=x\right\}} \pi(y)\right) \\
& =\frac{1}{2}\left(\pi(1+x)-\pi_{-}(1-x)\right)+\frac{1}{2}\left(\pi(\alpha+x)-\pi_{-}(\alpha-x)\right),
\end{aligned}
$$

where $\pi_{-}(y) \equiv \lim _{z \rightarrow y^{-}} \pi(z)$ (the limit exists since $\pi$ is increasing). We will only need to consider the points $\langle n \alpha\rangle$; Lemma 4.1 establishes that $\pi$ and $\pi_{-}$ are equal at these points. Recall, of course, that $\pi(x)=1$ for all $x \geqslant 1$ and $\pi(x)=0$ for all $x<0$.

Again we first treat the case $\frac{1}{2}<\alpha<1$. We already know $\pi(0)=0$ by Lemma 4.1, and also $\pi(1)=1$. Suppose $\pi(\alpha)=z \in[0,1]$. Evaluating (5) at $x=\alpha$, we get $\pi(\langle-\alpha\rangle)=2-2 z$. Similarly, at $x=\langle-\alpha\rangle$ we get $\pi(\langle 2 \alpha\rangle)=$ $3 z-2$, and so on. Using (5) repeatedly, we can compute $\pi(\langle n \alpha\rangle)$ for all $n \in \mathbf{Z}$. In particular, if $n>0$ and $\langle n \alpha\rangle$ is small, then we get $\pi(\langle n \alpha\rangle)=$ $\left(2 n-L_{n}\right) z-\left(2 n-2 L_{n}\right)$, where $L_{n}$ is the number of large vertices among $\langle\alpha\rangle, \ldots,\langle n \alpha\rangle$. Recall that $L_{n} / n \rightarrow 1-\alpha$.

We now claim that there is only one possible value of $z$. To see this, choose an increasing sequence of positive integers $\left\{n_{i}\right\}$ such that $\left\langle n_{i} \alpha\right\rangle \rightarrow 0$. We may assume the $\left\langle n_{i} \alpha\right\rangle$ are small. Since $\pi(0)=0$ and $\pi$ is right continuous, we have $0=\lim _{i \rightarrow \infty} \pi\left(\left\langle n_{i} \alpha\right\rangle\right)=\lim _{i \rightarrow \infty}\left[\left(2 n_{i}-L_{n_{i}}\right) z-\left(2 n_{i}-2 L_{n_{i}}\right)\right]$ whence $z=\lim _{i \rightarrow \infty}\left[\left(2 n_{i}-2 L_{n_{i}}\right) /\left(2 n_{i}-L_{n_{i}}\right)\right]=2 \alpha /(1+\alpha)$.

If $0<\alpha<\frac{1}{2}$ we proceed similarly and find that for $n>0$ and small $\langle n \alpha\rangle$, $\pi(\langle n \alpha\rangle)=\left(2 n-L M_{n}\right) z-\left(2 n-2 L M_{n}\right)$, where $L M_{n}$ is the number of large and medium vertices among $\langle\alpha\rangle, \ldots,\langle n \alpha\rangle$. We again have $L M_{n} / n \rightarrow 1-\alpha$, and the corresponding limiting argument again shows that $z=2 \alpha /(1+\alpha)$.

Note that by $(5), \pi_{\mu}(\alpha)=2 \alpha /(1+\alpha)$.

Theorem 4.1. Let $\mu$ be uniform on the set $\{\alpha, 1\}$, with $0<\alpha<1$ and $\alpha$ irrational. Let $\pi$ be a stationary distribution for the iterated random function. Then $\pi=\pi_{\mu}$.

$\mathrm{P}$ r o o f. Being right continuous, $\pi$ is determined by its values on the dense set $\mathscr{O}_{0}$. Therefore, by the above discussion, $\pi$ is determined by its 
value at $\alpha$. Finally, we have shown that there is only one possible value of $\pi(\alpha)$, namely the value $\pi_{\mu}(\alpha)$.

\section{Rate of convergence}

We will use the description of $G_{x}$ to give us a rate of convergence for the backwards iterates in Theorem 5.1. We shall need some preliminary results before proving the bound in Section 5.3.

5.1. Continued fractions. Fix $\alpha$ irrational, and let

$$
\alpha=a_{0}+\frac{1}{a_{1}+\frac{1}{a_{2}+\frac{1}{\ddots}}}
$$

be the continued fraction expansion of $\alpha$ (each $a_{i}$ is an integer, with $a_{i}>0$ if $i>0$ ). For $n \geqslant 0$ let $p_{n}$ and $q_{n}$ be relatively prime integers such that

$$
\frac{p_{n}}{q_{n}}=a_{0}+\frac{1}{a_{1}+\frac{1}{a_{2}+\frac{1}{\ddots \cdot+\frac{1}{a_{n}}}}} .
$$

It follows that $q_{n}$ satisfies the recursion $q_{n+1}=a_{n} q_{n}+q_{n-1}$ (see, e.g., [3]). The $p_{n}$ and $q_{n}$ are such that

$$
\left|\alpha-\frac{p_{n}}{q_{n}}\right|<\frac{1}{2 q_{n}^{2}}
$$

We shall make use of the following fact.

Lemma 5.1. For any $x \in[0,1]$, there is an integer $k$ with $0 \leqslant k<q_{n}$ such that $0 \leqslant\langle x-k \alpha\rangle<3 /\left(2 q_{n}\right)$.

P r o of. Let $S=\left\{\langle k \alpha\rangle: k=0,1, \ldots, q_{n}-1\right\}$, and for each $i=0,1, \ldots, q_{n}-1$ let $\bar{\imath}$ denote the unique integer among $0,1, \ldots, q_{n}-1$ satisfying $\bar{\imath} p_{n} \equiv i\left(\bmod q_{n}\right)$. Note that $0 \in S$. It essentially follows from $(8)$ that each of the $q_{n}-1$ (disjoint) intervals $\left(i / q_{n}-1 /\left(2 q_{n}\right), i / q_{n}+1 /\left(2 q_{n}\right)\right)$, where $i=1, \ldots, q_{n}-1$, contains exactly one point of $S$, namely $\langle\bar{\imath} a\rangle$. To see this, note that by (8),

$$
\left|\bar{\imath} \alpha-\bar{\imath} \frac{p_{n}}{q_{n}}\right|=\bar{\imath}\left|\alpha-\frac{p_{n}}{q_{n}}\right|<\bar{\imath} \cdot \frac{1}{2 q_{n}^{2}}<\frac{1}{2 q_{n}} .
$$

This implies that there is no integer strictly between $\bar{\imath} \alpha$ and $\bar{\imath} p_{n} / q_{n}$, and therefore $\left|\bar{\imath} \alpha-\bar{\imath} p_{n} / q_{n}\right|=\left|\langle\bar{\imath} \alpha\rangle-\left\langle\bar{\imath} p_{n} / q_{n}\right\rangle\right|$. Thus

$$
\left|\langle\bar{\imath} \alpha\rangle-\frac{i}{q_{n}}\right|=\left|\langle\bar{\imath} \alpha\rangle-\left\langle\bar{\imath} p_{n} q_{n}\right\rangle\right|=\left|\bar{\imath} \alpha-\frac{\bar{\imath} p_{n}}{q_{n}}\right|<\frac{1}{2 q_{n}} .
$$


Note also that if $\alpha>p_{n} / q_{n}$ then $\langle\bar{\imath} \alpha\rangle>i / q_{n}$ for all $i=0,1, \ldots, q_{n}-1$; whereas if $\alpha<p_{n} / q_{n}$ then $\langle\bar{\imath} \alpha\rangle<i / q_{n}$ for all such $i$. Thus no two consecutive points of $S$ are more than $3 /\left(2 q_{n}\right)$ apart.

To summarize, the smallest point of $S$ is 0 , any two consecutive points of $S$ are within $3 /\left(2 q_{n}\right)$ of each other, and the largest point of $S$ is greater than $\left(q_{n}-1\right) / q_{n}-1 /\left(2 q_{n}\right)=1-3 /\left(2 q_{n}\right)$. This proves the lemma.

5.2. Random walks on the line. Fix $0<\alpha<1$ irrational, and fix $0<x_{0}<\alpha$ in a generic orbit. The vertex $v_{0}$ corresponding to $x_{0}$ in the graph $G=G_{x_{0}}$ is small; thus (see Section 4.1) $v_{0}$ is a cut-vertex of $G$ (that is, $G-v_{0}$ is not connected).

For an arbitrary vertex $v$ of $G$, let $d\left(v_{0}, v\right)$ be the distance from $v_{0}$ to $v$ in $G$, ignoring orientations. Define a map $\rho$ from the vertex set of $G$ to $\mathbf{Z}$ as follows: let $\rho(v)=n>0$ if $d\left(v_{0}, v\right)=n$ and $v$ is in the right-hand component of $G-v_{0}$; let $\rho(v)=n<0$ if $d\left(v_{0}, v\right)=-n$ and $v$ is in the left-hand component of $G-v_{0}$; and let $\rho\left(v_{0}\right)=0$.

Observation. If $\theta$ is chosen uniformly from $\{\alpha, 1\}$ then for any $x \in \mathscr{O}_{x_{0}}$, we have

$$
\rho\left(f_{\theta}(x)\right)= \begin{cases}\rho(x)+1 & \text { with probability } \frac{1}{2} \\ \rho(x)-1 & \text { with probability } \frac{1}{2}\end{cases}
$$

The next lemma says that very long paths on $G$ must go through vertices which correspond to very small numbers. As $x_{0}$ is fixed, we no longer distinguish between a point $x \in \mathscr{O}_{x_{0}}$ and the corresponding vertex of $G$.

Lemma 5.2. Let $x, y \in \mathscr{O}_{x_{0}}$, and suppose that $\rho(x)-\rho(y) \geqslant 2 q_{m}$. Then there exists $z \in \mathscr{O}_{x_{0}}$ with $\rho(x) \geqslant \rho(z) \geqslant \rho(y)$ such that $0 \leqslant z<3 /\left(2 q_{m}\right)$.

P r o of. From the graph $G$ (more precisely, from the proof of Lemma 3.1), it is clear that for any $k \in \mathbf{Z}, k \leqslant|\rho(\langle x-k \alpha\rangle)-\rho(x)| \leqslant 2 k$.

Suppose first that $x=\left\langle r \alpha+x_{0}\right\rangle$ for some $r \in \mathbf{Z}$; thus the vertex $x$ is on the top row of the graph $G$ and $\langle x+\alpha\rangle$ is to the right of $x$. Lemma 5.1 gives a number $k$ with $0 \leqslant k<q_{m}$ and $0 \leqslant\langle x-k \alpha\rangle:=z<3 /\left(2 q_{m}\right)$. Then $\rho(y) \leqslant \rho(x)-2 q_{m} \leqslant \rho(z) \leqslant \rho(x)$ as desired.

On the other hand if $x$ is on the bottom row of $G$, i.e., $x=\left\langle r \alpha-x_{0}\right\rangle$ for some $r \in \mathbf{Z}$, then we apply Lemma 5.1 to the number $\left\langle x+\left(q_{m}-1\right) \alpha\right\rangle$ to get a $k$ with $0 \leqslant k<q_{m}$ and $0 \leqslant\langle x+k \alpha\rangle:=z<3 /\left(2 q_{m}\right)$. Again we have

$$
\rho(y) \leqslant \rho(x)-2 q_{m} \leqslant \rho(z) \leqslant \rho(x) .
$$

We shall need the following lemma; the simple proof provided was pointed out to us by D. Aldous.

Lemma 5.3. There exists an integer $N_{0}$ and a constant $c_{0}>0$ such that for all $n>N_{0}$, a simple random walk of length $n^{3}$ on a line remains within $n$ units of its starting point with probability less than or equal to $e^{-c_{0} n}$. 
P r o o f. For a walk to stay within $n$ units of its starting point it is necessary (though not sufficient) that no subwalk of the walk moves more than $2 n$ units in either direction. By the central limit theorem, there are an integer $N_{0}^{\prime}$ and a constant $c_{0}^{\prime}>0$ so that for $k>N_{0}^{\prime}$, a walk of length $c_{0}^{\prime} k^{2}$ remains within $k$ units of its starting point with probability less than $e^{-1}$. Given a walk of length $n^{3}$, we break it into pieces of length $c_{0}^{\prime} /\left(4 n^{2}\right)$ and apply the previous fact with $k=2 n$ to each of the $n /\left(4 c_{0}^{\prime}\right)$ pieces. It follows that the probability that the walk remains within $n$ units of its starting point is at most $e^{-n /\left(4 c_{0}^{\prime}\right)}$.

5.3. The bound. The content of the following theorem is essentially that with high probability, the entire interval $[0,1]$ gets within $\varepsilon$ of its limit under backward iteration after $O\left((1 / \varepsilon)^{3} \ln (1 / \varepsilon)\right)$ steps.

Theorem 5.1. Let $0<\alpha<1$ be irrational, and fix $\varepsilon$ small and positive. Find $k$ such that $8 / q_{k}<\varepsilon$ (where $q_{k}$ is as in Section 5.1), and let $N=8 q_{k}^{3} \log _{2} q_{k}$. There is a constant $c>0$, which does not depend on $\varepsilon$, such that with probability larger than $1-e^{-c / \varepsilon}$, we have

$$
\left|B_{n}(\Theta, x)-B(\Theta)\right|<\varepsilon \text { for all } n \geqslant N \text { and for all } x \in[0,1],
$$

where $\Theta=\left(\theta_{1}, \theta_{2}, \ldots\right)$ is chosen uniformly from $\{\alpha, 1\}^{\infty}$.

P r o o f. Let $j=8 q_{k}^{3}$. Given an interval $I$ we compute a lower bound $\mathrm{p}$ for the probability that

$$
\left\|f_{\theta_{1}} \circ \cdots \circ f_{\theta_{j}}(I)\right\| \leqslant \frac{1}{2}\|I\|+\frac{2}{q_{k}} .
$$

It follows then that with probability bigger than $\mathrm{p}^{t},\left\|f_{\theta_{1}} \circ \cdots \circ f_{\theta_{t j}}([0,1])\right\| \leqslant$ $(1 / 2)^{t}+4 / q_{k}$. In this way, with $t=\left\lfloor\log _{1 / 2}\left(4 / q_{k}\right)+1\right\rfloor=\left\lfloor\log _{2} q_{k}-1\right\rfloor$ and $n \geqslant t j$ fixed, we have that for all $x \in[0,1]$, (9) holds, i.e.,

$$
\begin{aligned}
& \left|B_{n}(\Theta, x)-B(\Theta)\right|=\lim _{m \rightarrow \infty}\left|B_{n}(\Theta, x)-f_{\theta_{1}} \circ \cdots \circ f_{\theta_{m}}(x)\right| \\
& \quad=\lim _{m \rightarrow \infty}\left|B_{n}(\Theta, x)-B_{n}\left(\Theta, f_{\theta_{n+1}} \circ \cdots \circ f_{\theta_{m}}(x)\right)\right|<\frac{8}{q_{k}}<\varepsilon,
\end{aligned}
$$

with probability at least $\mathrm{p}^{t}$.

We get the estimate $\mathrm{p}$ as follows. Pick a point $m^{*} \in \mathscr{O}_{x_{0}}\left(x_{0}\right.$ as in Section 5.2) such that $m^{*}$ is within $\delta=1 /\left(2 q_{k}\right)$ of the midpoint of $I$ (recall that $\mathscr{O}_{x_{0}}$ is dense). By the observation in Section 5.2, the sequence

$$
\rho\left(m^{*}\right), \rho\left(f_{\theta_{j}}\left(m^{*}\right)\right), \rho\left(f_{\theta_{j-1}} \circ f_{\theta_{j}}\left(m^{*}\right)\right), \ldots, \rho\left(f_{\theta_{1}} \circ \cdots \circ f_{\theta_{j}}\left(m^{*}\right)\right)
$$

is a simple random walk on $\mathbf{Z}$.

If this walk does not stay within $2 q_{k}$ units of $\rho\left(m^{*}\right)$ on the line, then we can apply Lemma 5.2 to get a $j_{0}, 1 \leqslant j_{0} \leqslant j$, such that $f_{\theta_{j_{0}}} \circ \cdots \circ f_{\theta_{j}}\left(m^{*}\right)<$ 
$3 /\left(2 q_{k}\right)$. Then Lemma 3.2 implies that $\left\|f_{\theta_{j_{0}}} \circ \cdots \circ f_{\theta_{j}}(I)\right\| \leqslant\|I\| / 2+\delta+$ $3 /\left(2 q_{k}\right) \leqslant\|I\| / 2+2 / q_{k}$. Since $f_{\theta}$ never increases the length of an interval, (10) holds.

With $c_{0}$ and $N_{0}$ as in Lemma 5.3, we therefore have $\mathrm{p} \geqslant 1-e^{-2 c_{0} q_{k}}$ (provided that $2 q_{k}>N_{0}$ ). Thus for $n \geqslant 8 q_{k}^{3} \log _{2} q_{k} \geqslant t j$ we have that (9) holds with probability at least $\left(1-e^{-2 c_{0} q_{k}}\right)^{\log _{2} q_{k}}$. By the binomial theorem, this last expression is the same as

$$
1-\frac{\log _{2} q_{k}}{e^{2 c_{0} q_{k}}}+O\left(\frac{\log _{2}^{2} q_{k}}{e^{4 c_{0} q_{k}}}\right)
$$

which, for large $q_{k}$, is at least $1-c^{\prime} e^{-2 c_{0} q_{k}} \log _{2} q_{k}>1-e^{-c q_{k}}$. This is the desired result.

As an application of this theorem, let $\alpha=1 / \sqrt{2}$. Here (see Section 5.1) we have $a_{0}=0, a_{1}=1$, and $a_{i}=2$ for $i \geqslant 2$. The recurrence relation for $q_{n}$ implies that $q_{n}=\frac{1}{2}\left((1+\sqrt{2})^{n}+(1-\sqrt{2})^{n}\right)$. The theorem implies in this case that there are constants $c$ and $C$ such that for $n \geqslant C \varepsilon^{-3} \ln (1 / \varepsilon)$, and for all $x \in[0,1]$, the $n$-th backward iterate is within $\varepsilon$ of its limit with probability at least $1-e^{-c / \varepsilon}$. The same statement holds for any $\alpha$ for which the set of $a_{i}$ 's (see (7)) is bounded; in particular this holds for any quadratic irrational $\alpha$.

Acknowledgments. We thank Persi Diaconis for suggesting this problem and for numerous helpful conversations. We also thank Julie Landau, without whose support and net game this paper would not have been written.

\section{REFERENCES}

1. Diaconis P., Freedman D. Iterated random functions. - SIAM Rev., 1999, v. 41, № 1, p. $45-76$.

2. Feller $W$., An Introduction to Probability Theory and Its Applications. V. II. New York: Wiley, 1971, 669 p.

3. Hardy G. H., Wright E. M. An Introduction to the Theory of Numbers. Oxford: Clarendon Press, 1979, 426 p.

4. Letac G. A contraction principle for certain Markov chains and its applications. Contemp. Math., 1986, v. 50, p. 263-273.

5. Mattingly J.C. Contractivity and ergodicity of the random map $x \mapsto|x-\theta|$. Теория вероятн. и ее примен., 2002, т. 47, в. 2, с. 388-397.

Поступила в редакцию 22.XI.2001 